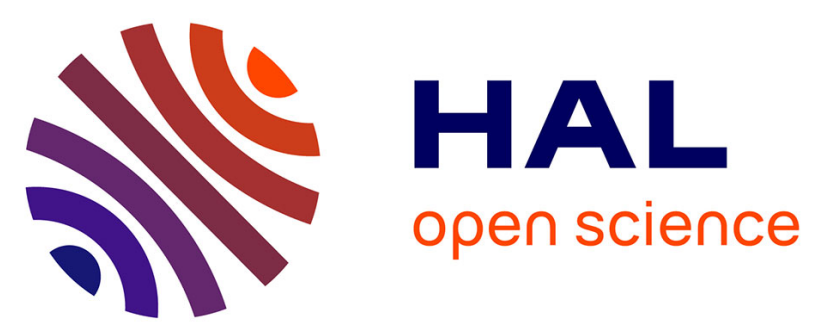

\title{
Structure accélératrice de symétrie plane à auges rectangulaires
}

\author{
M. Boussoukaya, Nguyen Tuong Viet, A. Septier
}

\section{To cite this version:}

M. Boussoukaya, Nguyen Tuong Viet, A. Septier. Structure accélératrice de symétrie plane à auges rectangulaires. Journal de Physique Lettres, 1976, 37 (11), pp.317-320. 10.1051/jphyslet:019760037011031700 . jpa-00231301

\section{HAL Id: jpa-00231301 https://hal.science/jpa-00231301}

Submitted on 1 Jan 1976

HAL is a multi-disciplinary open access archive for the deposit and dissemination of scientific research documents, whether they are published or not. The documents may come from teaching and research institutions in France or abroad, or from public or private research centers.
L'archive ouverte pluridisciplinaire HAL, est destinée au dépôt et à la diffusion de documents scientifiques de niveau recherche, publiés ou non, émanant des établissements d'enseignement et de recherche français ou étrangers, des laboratoires publics ou privés. 


\title{
STRUCTURE ACCÉLÉRATRICE DE SYMÉTRIE PLANE A AUGES RECTANGULAIRES

\author{
M. BOUSSOUKAYA, NGUYEN TUONG VIET
}

\author{
Institut d'Electronique Fondamentale $\left(^{*}\right)$ \\ Université Paris XI, Bâtiment 220, 91405 Orsay Cedex, France
}

et A. SEPTIER

C.N.A.M., 292, rue Saint-Martin, 75141 Paris Cedex 03, France

(Reçu le 19 mai 1976, accepté le 28 juillet 1976)

\begin{abstract}
Résumé. - Les valeurs des fréquences de résonances, du coefficient de surtension $Q_{0}$ pour différentes configurations du mode accélérateur - et du mode déflecteur — ont été mesurées sur un prototype de cavité à 6 auges rectangulaires à symétrie plane fonctionnant en bande $\mathrm{S}$. Les meilleures performances sont atteintes lorsque la structure est ouverte sur les côtés latéraux, et résonne sur le mode $\pi / 2$.
\end{abstract}

\begin{abstract}
Using a six cell periodic plane symmetric structure, values of resonant frequencies and the quality factor $Q_{0}$ were measured for different field configurations of the accelerating and deflecting modes in the S-band. Best results were obtained for a structure which is open along its sides, resonating in the $\pi / 2$ mode.
\end{abstract}

1. Introduction. - Les guides circulaires à iris utilisés comme structures à ondes lents dans des accélérateurs linéaires supraconducteurs sont réalisés par assemblage et soudure par bombardement électronique, d'un grand nombre de cellules élémentaires en Niobium. Cette technique est délicate et coûteuse. De plus, il est très difficile de contrôler l'état de surface des parois internes après polissage ou oxydation contrôlée.

C'est pourquoi la structure accélératrice à symétrie plane proposée récemment et utilisée à l'Université Cornell $[1,2,3]$ présente, au point de vue technologique, de multiples avantages : il est possible d'usiner de grandes longueurs de guide dans des plaques épaisses de niobium, et d'effectuer aisément les traitements et contrôles d'état de surface.

Nous ne disposons encore actuellement que de peu de données sur les propriétés de cette structure. C'est pourquoi nous avons entrepris une étude sur un prototype relativement court, réalisé en laiton et fonctionnant à la température ambiante, afin d'en déterminer les caractéristiques électromagnétiques essentielles, en vue d'une comparaison avec celles du guide à iris classique.

(*) Laboratoire associé au C.N.R.S.
2. Description. - La cavité est formée de deux séries identiques d'auges parallélépipédiques $\left(76,2 \times 47,5 \times 25 \mathrm{~mm}^{3}\right)$ accolées, obtenues par fraisage dans deux plaques identiques de laiton épaisses de $30 \mathrm{~mm}$, de longueur $400 \mathrm{~mm}$ et de largeur $160 \mathrm{~mm}$. Les auges sont séparées par des parois de $5 \mathrm{~mm}$ d'épaisseur, jouant un rôle identique à celui des iris du guide circulaire. La figure 1 en donne deux vues schématiques. L'axe de la cavité $O z$ est dirigé suivant la longueur des plaques rectangulaires.

Les plaques sont placées face à face, à une distance $e=25 \mathrm{~mm}$. L'écartement est assuré, dans la première configuration étudiée, par un cadre rectangulaire de laiton à section carrée $\left(25 \times 25 \mathrm{~mm}^{2}\right)$ dont la face interne est située à la distance $d$ du bord externe des auges. Les deux grandeurs $e$ et $d$ peuvent varier par usinage du cadre.

Après les premières expériences sur cette structure entièrement fermée, on a successivement supprimé les parties latérales du cadre, puis les parties situées sur le trajet de l'axe $O z$, obtenant ainsi une structure partiellement - ou totalement - ouverte. L'écartement entre les deux parties est suffisamment faible pour que les champs électromagnétiques excités dans la région des auges deviennent évanescents entre les plans métalliques entourant ces auges.

La cavité est associée à un générateur UHF et à 


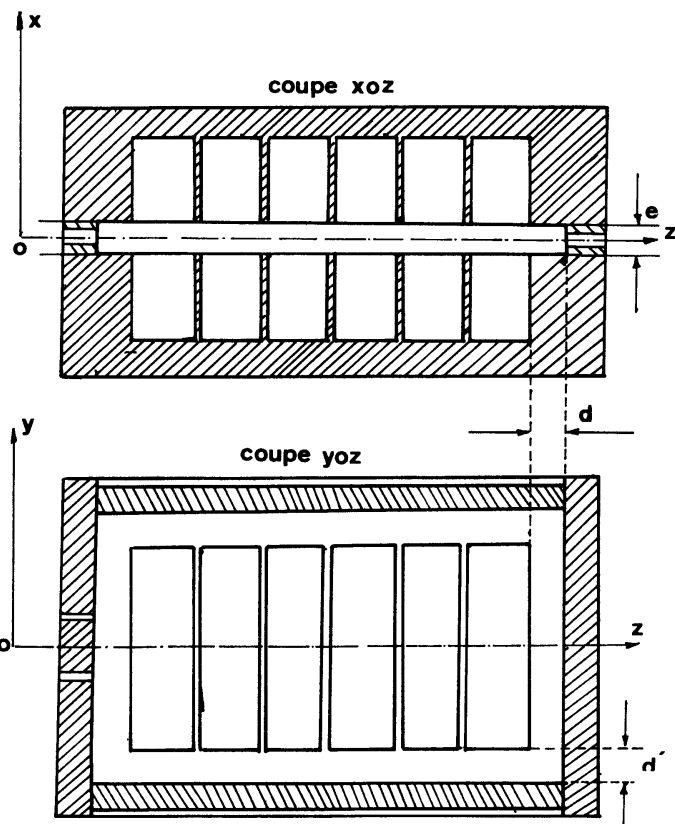

FIG. 1. - Vue schématique de la structure à six auges : a) coupe $x O z$; b) coupe $y O z$

une diode détectrice grâce à deux sondes de petites dimensions très faiblement couplées, excitant ou détectant le champ électrique longitudinal $E_{z}$ dans le plan de symétrie $x \mathrm{Oz}$.

3. Propriétés du mode accélérateur. - Le mode accélérateur correspond à la configuration $\mathrm{E}_{110}$ des cavités rectangulaires élémentaires obtenues en faisant $e=0$. Transformée en cavité résonnante, une telle structure périodique à 6 cellules élémentaires résonne sur plusieurs fréquences situées dans la bande passante du mode accélérateur. Chacune correspond à un déphasage différent de l'onde à la traversée d'une cellule, égal à $n \frac{\pi}{6}$ ( $n$ entier) tel que $0<n<6$, et la configuration de champ obtenue pour chacune de ces fréquences est appelée improprement mode $n \frac{\pi}{6}$.

Il n'existe actuellement aucune méthode permettant un calcul précis des fréquences de résonance d'une telle cavité. Nous nous sommes inspirés des modèles présentés schématiquement dans les références [1] et [2]. Un calcul approché de la fréquence de résonance du système résonnant sur le mode $\pi$ peut être effectué pour l'une des cavités élémentaires formées par deux auges placées face à face, et séparées par la distance $e$, excitées sur le mode $\mathrm{E}_{110}$. On obtient un parallélipipède de dimensions $76,2 \mathrm{~mm}$ (suivant $O x$ ), $75 \mathrm{~mm}$ (suivant $O y$ ). La fréquence de résonance ne dépend pas de la cote suivant $O z$. On obtient $f_{0}=2806 \mathrm{MHz}$, alors que l'expérience (voir plus loin) donne $2810 \mathrm{MHz}$ pour l'ensemble de la structure.

Une méthode classique de perturbation de la fréquence de résonance par un obstacle de très petit volume permet d'obtenir la répartition du champ électrique de l'onde stationnaire $\left[{ }^{4}\right]$, soit sur l'axe $O z$, soit dans une direction transversale. La perturbation de fréquence de résonance $\Delta f_{0} / f_{0}$ par une fine aiguille diélectrique $\left(0,5 \times 8 \mathrm{~mm}^{2}\right)$ est en effet proportionnelle au carré de l'amplitude de la composante du champ électrique parallèle à l'aiguille. Lorsque le champ magnétique HF est nul - et c'est le cas pour le mode accélérateur lorsqu'on déplace l'obstacle suivant l'axe $\mathrm{Oz}$ - on peut utiliser une petite sphère métallique ; la variation de fréquence $\Delta f_{0} / f_{0}$ est encore proportionnelle à $E^{2}$, et peut être calculée avec précision :

$$
\Delta f_{0} / f_{0}=\frac{3}{2} v \varepsilon_{0} E^{2} / W
$$

( $v=$ volume de la sphère, $W=$ énergie totale emmagasinée dans la cavité). Un signal électrique proportionnel à $\Delta f_{0} / f_{0}$ est envoyé en ordonnées sur une table traçante, les abscisses étant proportionnelles au déplacement de l'obstacle. On a relevé ainsi les courbes de variation de $E_{z}^{2}$ suivant $O z$ pour les différentes fréquences de résonance, ce qui a permis l'identification des modes et la détermination de la longueur d'onde $\lambda_{\mathrm{g}}$ des ondes se propageant dans la structure. Les figures $2(a, b, c)$ montrent la variation de champ $E_{z}^{2}$ pour le mode $\pi / 2$ dans la cavité, lorsque celle-ci est fermée, ouverte latéralement, ou totalement ouverte. Les figures $3(a, b)$ correspondent à la répartition $\mathrm{du}$ mode $\pi$.

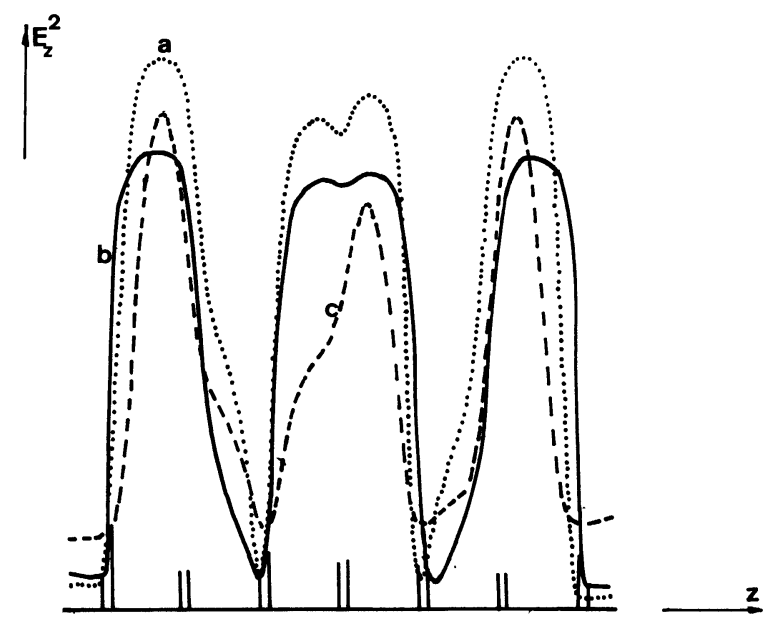

FIG. 2. - Distribution de $E_{z}^{2}$ pour le mode $\pi / 2$ dans la structure : a) fermée; $b$ ) ouverte latéralement ; $c$ ) totalement ouverte.

La décroissance de $E_{z}$ dans les auges extrêmes s'explique par le couplage avec la zone de champ évanescent. En retouchant les dimensions des cellules extrêmes, il doit être possible d'obtenir $E_{z}$ constant d'un bout à l'autre du guide.

Dans notre cavité, le mode $n=0$ ne peut être observé, car il exige la présence de plans réfléchissants pour $E_{z}$, situés au milieu des auges extrêmes ou au milieu des iris.

La courbe de dispersion s'étend de $2702 \mathrm{MHz}$ (mode $\pi / 6$ ) à $2810 \mathrm{MHz}(\operatorname{mode} \pi)$ pour $e=25 \mathrm{~mm}$ et $d=7 \mathrm{~mm}$. 


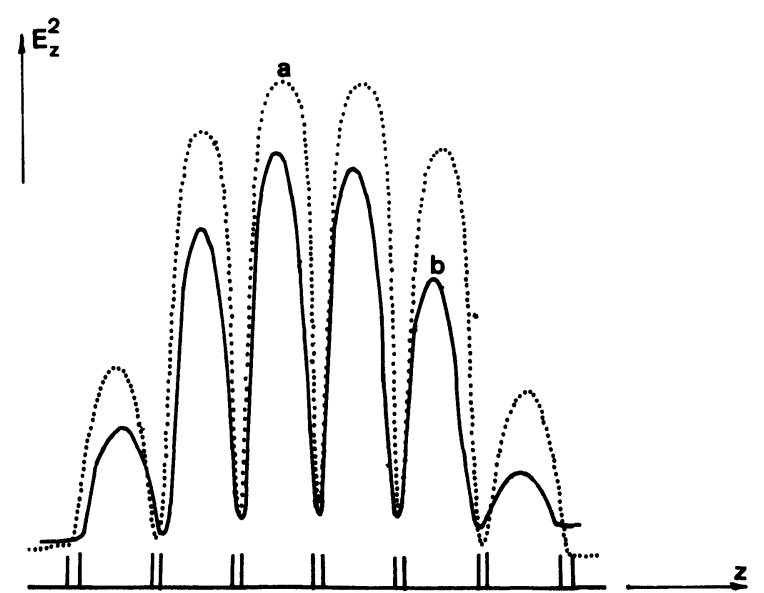

FIG. 3. - Répartition de $E_{z}^{2}$ pour le mode $\pi: a$ ) cavité fermée ; b) cavité ouverte latéralement.

Nous avons en outre pour chaque mode mesuré la valeur du coefficient de surtension $Q$, pratiquement égal au coefficient de surtension à vide $Q_{0}$, par suite de la valeur très faible des couplages avec les circuits extérieurs.

Le tableau I donne le coefficient de surtension $Q_{0}$, et la fréquence de résonance, pour les modes $n \frac{\pi}{6}$ dans les trois configurations successivement étudiées : cavité entièrement fermée $(e=25 \mathrm{~mm}, d=7 \mathrm{~mm})$, cavité ouverte sur les côtés latéraux $(e=25 \mathrm{~mm}$, $d=7 \mathrm{~mm}$ aux extrémités), puis cavité totalement ouverte $(e=25 \mathrm{~mm})$.

On voit que pour la cavité fermée, $Q_{0}$ est maximum pour le mode $\pi / 2$. Les pertes diminuent très nettement lorsqu'on enlève les parois latérales $\left(Q_{0} \simeq 8000\right)$, mais sont considérablement accrues lorsque la cavité est ouverte sur ses quatre côtés. Dans ce dernier cas, $Q_{0}$ est le plus élevé pour les modes extrêmes $=\pi / 6$ et $\pi$.

Le tableau II montre l'évolution des fréquences de résonance $f_{0}$ et de $Q_{0}$ pour la structure ouverte latéralement résonnant sur le mode $\pi / 2$, lorsqu'on fait varier la distance $d$ séparant le bord des auges extrêmes des plans réfléchissants mobiles placés normalement à $O x$, pour $e=25 \mathrm{~mm}$.

La distance d'optimum se situe vers $d=12 \mathrm{~mm}$.

Enfin le tableau III donne les valeurs de $f_{0}$ et $Q_{0}$ obtenues pour $d=7 \mathrm{~mm}$, lorsqu'on fait varier l'écartement $e$ des deux plaques, la cavité étant ouverte latéralement, et résonnant sur le mode $\pi / 2$.

L'écartement optimum est de l'ordre de $20 \mathrm{~mm}$.

Pour le mode $\pi$, la vitesse de phase $v_{\varphi}$ de l'onde est proche de $c$, et la vitesse de groupe nulle. Le mode $\pi / 2$ dans cette cavité particulière est tel que $v_{\varphi} \simeq 2 c$ et $v_{\mathrm{g}} \simeq 0,85 \mathrm{c}$.

A partir des valeurs de $f_{0}, Q_{0}$, de la perturbation de fréquence $\Delta f_{0} / f_{0}$ provoquée par une bille métallique de volume $v$, il est possible de calculer, dans une cavité de longueur $L$, une valeur approchée de l'impédance shunt du mode accélérateur, par la relation valable pour le guide à onde progressive :

$$
Z_{\mathrm{s}}=\frac{L}{3 \pi} \cdot \frac{\Delta f_{0}}{f_{0}} \cdot \frac{Q_{0}}{\varepsilon_{0} f_{0}}\left(\Omega \mathrm{m}^{-1}\right)
$$

On obtient respectivement pour le mode $\pi$, $Z_{\mathrm{s}(\pi)} \simeq 20 \mathrm{M} \Omega \mathrm{m}^{-1}$ et pour le mode $\pi / 2$,

$$
Z_{\mathrm{s}(\pi / 2)} \simeq 22,5 \mathrm{M} \Omega \mathrm{m}^{-1}
$$

dans la cavité en laiton ouverte latéralement. Pour une structure réalisée en cuivre OFHC, dont la

TABLEAU I

\begin{tabular}{|c|c|c|c|c|c|c|}
\hline \multirow{2}{*}{$\begin{array}{c}\text { Mode } \\
\text { accélérateur }\end{array}$} & \multicolumn{2}{|c|}{ Cavité fermée } & \multicolumn{2}{|c|}{$\begin{array}{c}\text { Cavité ouverte } \\
\text { latéralement }\end{array}$} & \multicolumn{2}{|c|}{$\begin{array}{c}\text { Cavité ouverte } \\
\text { totalement }\end{array}$} \\
\hline & $Q_{0}$ & $f_{0}(\mathrm{MHz})$ & $Q_{0}$ & $f_{0}(\mathrm{MHz})$ & $Q_{0}$ & $f_{0}(\mathrm{MHz})$ \\
\hline - & - & - & - & - & - & - \\
\hline$\pi / 6$ & 5560 & 2702 & 6685 & 2698 & 2290 & 2669 \\
\hline$\pi / 3$ & 4850 & 2714 & 6610 & 2709 & 560 & 2688 \\
\hline$\pi / 2$ & 6530 & 2738 & 8000 & 2736 & 485 & 2716 \\
\hline $2 \pi / 3$ & 5325 & 2763 & 5520 & 2762 & 480 & 2749 \\
\hline $5 \pi / 6$ & 4180 & 2790 & 4215 & 2788 & 780 & 2771 \\
\hline$\pi$ & 6050 & 2810 & 6100 & 2809 & 2285 & 2798 \\
\hline
\end{tabular}

Fréquences de résonance et coefficient de surtension de la cavité pour $e=25 \mathrm{~mm}, d=7 \mathrm{~mm}$.

\section{TABLEAU II}

Variation de $f_{0}$ et $Q_{0}$ dans la cavité ouverte latéralement, résonnant sur le mode $\pi / 2$. $d$ est la distance entre les plans de court-circuit et les bords des auges d'extrémité. $e=25 \mathrm{~mm}$

$\begin{array}{lrrrrrr}d(\mathrm{~mm}) & 2 & 7 & 12 & 17 & 22 \\ Q_{0} & 7350 & 8000 & 8055 & 5590 & 5400 \\ f_{0}(\mathrm{MHz}) & 2739 & 2736 & 2734 & 2733 & 2730\end{array}$

Variation de $f_{0}$ et $Q_{0}$ dans la cavité ouverte latéralement résonnant sur le mode $\pi / 2$ en fonction de $e$, pour $d=7 \mathrm{~mm}$

$\begin{array}{lrrrrr}e(\mathrm{~mm}) & 10 & 15 & 20 & 25 & 30 \\ Q_{0} & 8285 & 8330 & 8370 & 8000 & 7665 \\ f_{0}(\mathrm{MHz}) & 2785 & 2757 & 2744 & 2736 & 2720\end{array}$


résistance superficielle est au moins 2 fois plus faible que celle de notre laiton, ces valeurs seraient de l'ordre de 45 à $50 \mathrm{M} \Omega / \mathrm{m}$, c'est-à-dire légèrement inférieures, mais comparables à celles d'un guide accélérateur à iris circulaires $(50$ à $60 \mathrm{M} \Omega / \mathrm{m}$ à la température ambiante).

4. Mode déflecteur. - On nomme ainsi des modes ayant, dans le plan de symétrie $x O z$, la composante de champ électrique dirigée suivant $O y$. Grâce à l'emploi d'une aiguille diélectrique déplacée dans les auges suivant des parallèles à $O y$, puis à $O z$, il est possible d'identifier un tel mode. Nous avons déterminé la bande passante, et les répartitions de $E_{y}$ suivant $O z$ pour les différents modes $m \frac{\pi}{6}$ du mode déflecteur fondamental. Les fréquences de résonance sont comprises entre 2839 et $2903 \mathrm{MHz}$. La caractéristique de dispersion est directe (vitesse de groupe positive) alors que, dans le guide à iris classique, elle est le plus souvent inverse. Le tableau IV en donne les principales caractéristiques, pour $e=20 \mathrm{~mm}$ et $d=7 \mathrm{~mm}$.

Nous constatons d'une part que le mode $\pi / 2$ donne un coefficient de surtension supérieur à celui du $\operatorname{mode} \pi$,
TABleaU IV

Mode déflecteur. Cavité ouverte latéralement. $e=25 \mathrm{~mm}, d=7 \mathrm{~mm}$

$\begin{array}{lcccccc}\text { Mode } & \pi / 6 & \pi / 3 & \pi / 2 & 2 \pi / 3 & 5 \pi / 6 & \pi \\ - & - & - & - & - & - & - \\ Q_{0} & 6090 & 6030 & 6390 & 6185 & 5050 & 5060 \\ f_{0} & 2839 & 2848 & 2861 & 2877 & 2892 & 2903 \\ (\mathrm{MHz}) & & & & & & \end{array}$

d'autre part que les fréquences de résonance du mode déflecteur sont proches de celles du mode accélérateur (œ qui n'est pas le cas dans un guide à iris où $f$ (mode déflecteur) $\simeq 1,5$ (mode accélérateur).

5. Conclusion. - Le guide à auges rectangulaires placées face à face à une distance de $20 \mathrm{~mm}$, ouvert sur les côtés latéraux, possède des propriétés comparables à celles du guide à iris circulaire. Les résultats préliminaires obtenus au cours de cette étude vont permettre de construire un second prototype fonctionnant sur le mode $\pi / 2$ à $3000 \mathrm{MHz}$, et tel que la vitesse de phase de l'onde accélératrice soit égale à $c$ pour ce mode, puis une cavité accélératrice en niobium.

\section{Bibliographie}

[1] Sundelin, R., Von Borstel, E., Kirchgessner, J., Rice, D., Tigner, M., IEEE Trans. Nucl. Sci. 20 (1973) 98.

[2] Sundelin, R., Kirchgessner, J., Padamsee, H., Phillips, H. L., Rice, D., Tigner, M., Von Borstel, E., Proc. 9th Conf. High Energy Accelerator, SLAC (mai 1974) 128.
[3] Kirchgessner, J., Padamsee, H., Phillips, H. L., Rice, D., Sundelin, R., Tigner, M., Von Borstel, E., Proc. Int. Acc. Conf., Washington, IEEE Trans. Nucl. Sci. 22 (1975) 141.

[4] Maier, L. C., Slater, J. C., J. Appl. Phys. 23 (1952) 68. 